\title{
Simulation and Performance Evaluation of Energy-Efficient Routing Protocol in self-organised MANET's
}

\author{
P.P.Kotekar ${ }^{1}$, Prof. D.G.Chougule ${ }^{2}$ \\ Student, Department of Electronics Engineering, T.K.I.E.T, Warananagar, Maharashtra, India ${ }^{1}$ \\ Associate Professor, Department of Electronics Engineering, T.K.I.E.T, Warananagar, Maharashtra, India ${ }^{2}$
}

\begin{abstract}
Mobile ad hoc network is a collection of wireless node and this network is self-organising and configures multi-hop network. Basically each mobile node will be operated on battery. So for transmission and reception it will consume the nodes energy. Because of limited power it will drain faster. So to provide energy-efficient route between source and destination, the routing protocol must consider the residual energy of the node. In this paper, we first introduce an energy model to calculate the energy of the node than we propose the energy-efficient routing protocol by modifying the packet structure of AODV protocol. The performance of proposed protocol is analysed by using performance metrics that is routing overhead, energy consumption, end to end delay and packet delivery ratio.
\end{abstract}

Keywords: mobile ad hoc network, multi-hop, AODV, unicast.

\section{INTRODUCTION}

MANET is wireless network which is collection of by considering the node's remaining power. So the wireless mobile node and form a temporary network remaining power is used to choose the next hop. To without aid of any established infrastructure or centralized minimize the energy consumption of the mobile wireless administration. Ad Hoc wireless network has applications networks, the distributed position based protocols in emergency search and-rescue operations, decision optimization techniques are illustrated in [4] and [5] and making in the battlefield, data acquisition operations in the authors [6] has been proposed new Blocking-ERS hostile terrain, etc. It is featured by dynamic topology approach for demonstrating the substantial energy(insfrastructrureless), multi-hop communication; limited efficient Expanding Ring Search methods. In [7], to resources (bandwidth, CPU, battery, etc.) and limited measure the energy consumption of an IEEE 802.11 security. These characteristics put special challenges in wireless ad hoc network a series of experiments have been routing protocol design.

The primary objectives of MANET routing protocols are to maximize network throughput, to maximize network lifetime, and to maximize delay. The network throughput is usually measured by packet delivery ratio while the most significant contribution to energy consumption is measured by routing overhead which is the number or size of routing control packets. A major challenge that a routing protocol designed for ad hoc wireless networks faces is resource constraints. Devices used in the ad hoc wireless networks in most cases require portability and hence they also have size and weight constraints along with the restrictions on the power source.

In [1], the authors have been classified the energy consumption in MANET's in two ways: the active communication energy and inactive communication energy consumption where the energy is going to be consumed. In active communication energy is consumed by the reception and transmission of the packets and in inactive energy consumption, the node is idle but listen to the wireless medium. In [2], enhanced AODV routing protocol have been proposed in for improving the MANET lifetime. The authors of [3] said that sometimes the shortest route has been selected between source and destination path but every time it is not the best path. So they have proposed the enhanced -AODV routing protocol described.

In [8], to balance the energy consumption of individual node, a genetic algorithm based energy entropy multipath routing scheme has been proposed; find the minimal node's residual energy of each route, and prolong the lifetime and energy variance of the network. The performance improvement techniques of routing protocols have been presented in [9] and [10] for gateway interconnection and route reversal by considering energy consumption of the nodes. In these work, the energyefficient routing protocols have been designed to improve the performance in terms of end-to-end delay, and packet delivery ratio of the networks. On the other hand, an energy conserving mechanism is suggested in [11] to reduce the energy expenditure due to overhearing in MANETs by using a probability based overhearing node selection technique integrated with AODV protocol to minimize energy consumption of the networks.

\section{Proposed Modelling}

Energy-consumption of a node not only affects the lifetime of the node but also its ability to forward packets to other nodes. Thus, it affects the overall network lifetime and performance. It is a well known fact that the insufficient power of a node can lead to link failures in the constructed route and requires re-establishment procedure. 
So in order to overcome these disadvantages, we have proposed the energy model to measure the minimum energy factor $(\mathrm{EFm})$ of the node. Energy factor is the ratio of residual energy to the initial energy and also we have defined threshold value (Th-EF) for the energy-factor.

Most of the existing routing protocols such as AODV (Ad hoc on demand distance vector routing protocol) do not considered the nodal energy for preparing routing table. For that we have modified the existing AODV protocol by adding the extra field named as Minimum-Energy Factor (EFm) calculated by energy model and also we are defining the threshold value of the Energy-Factor (Th-EF) to establish an energy-efficient route from source to destination. We modified existing AODV protocol and named it as Protocol for Energy-Efficient Routing (PEER). In this, modified protocol that is PEER improves the performance and optimizes the energy consumption in MANET's.

To recommend the modified AODV protocol for MANET's by evaluating the performance in terms of as follows:

- $\quad$ Routing Overhead

- Energy Consumption per Packet

- End to End Delay

- Packet Delivery Ratio

\section{III.SCOPE OF WORK}

To design energy-efficient routing protocol, we first describe an energy model to measure the energy-factor of the nodes and then we propose a trust based Protocol for Energy-Efficient Routing (we name it as PEER). The partial mechanism of RREQ (Route Request) broadcast of existing AODV can be understood from ad hoc network. Suppose MANET network consisting of 49 nodes $\&$ has to send packet from source node 49 and destination node 7 . This network is of ad hoc wireless network into which Network layer protocol is AODV and Datalink and Physical layer is of 802.11 wlan. According to AODV protocol RREQ Message will be broadcasted from source node 49 for searching destination node 7 . This broadcasted message will again be broadcasted further by intermediate nodes in search of destination node 7 . For RREQ message appropriate destination node 7 will only send RREP (Route Reply) to the source node 49 which is of unicast type.

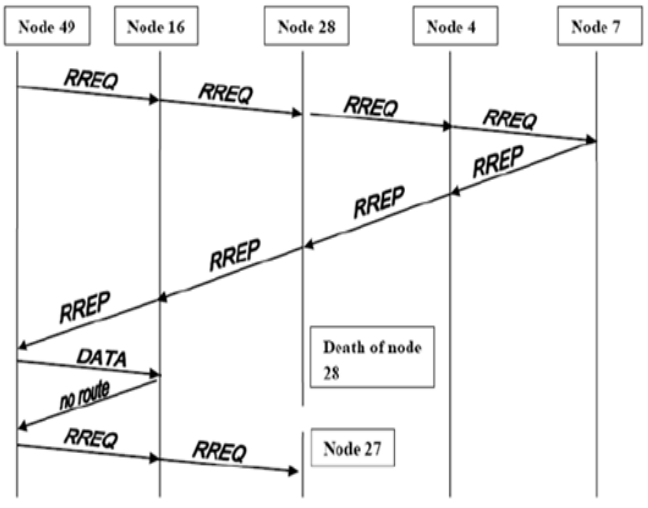

Fig. 1: Basic ADOV message 
International Journal of Advanced Research in Computer and Communication Engineering Vol. 4, Issue 5, May 2015

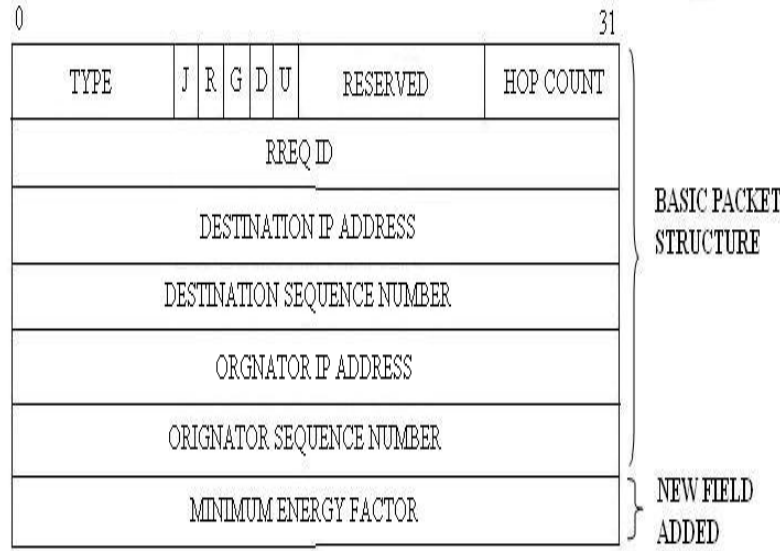

Fig. 3: Modified packet structure for RREQ

If the destination node has received more than one RREQ packets with equal values of EFm, then the destination node calculates the ratio of EFm and hop counts. Finally, it selects maximum value of ratios to unicast RREP packet. Source immediately starts to send data packets after receiving RREP.

\begin{tabular}{|c|c|c|c|c|}
\hline TYPE & R $A$ & RESERVED & PREFXXSIZE & HOP COONI \\
\hline \multicolumn{5}{|c|}{ DESTINATION P ADDRESS } \\
\hline \multicolumn{5}{|c|}{ DESTINATION SEQUENCENTOMBER } \\
\hline \multicolumn{5}{|c|}{ ORIGNATOR P ADDRESS } \\
\hline \multicolumn{5}{|c|}{ LFETIE } \\
\hline \multicolumn{5}{|c|}{ MINMOM ENERGY FACTOR } \\
\hline
\end{tabular}

BASIC PACKEI STRUCTURE

Fig. 4: Modified packet structure for RREP

\section{RESUlts}

We have used Network Simulator-2 for simulating the Proposed Protocol along with the AODV protocol. Table I shows the parameters being considered while simulation.

TABLE I

SIMULATION PARAMETERS

\begin{tabular}{|l|l|}
\hline \multicolumn{1}{|c|}{ Parameters } & \multicolumn{1}{c|}{ Values } \\
\hline Network Area & $1200 \mathrm{~mm} \times 12000 \mathrm{~mm}$ \\
\hline Packet Size & 70 bytes \\
\hline Maximum No. of Nodes & 50 \\
\hline Bandwidth & $2 \mathrm{Mbps}$ \\
\hline Simulation Time & 100 seconds \\
\hline
\end{tabular}

We have compared the performance of proposed protocol that is modified AODV (PEER) with AODV protocol in terms of Routing overhead, Energy consumption per packet, End to End Delay and Packet Delivery Ratio and by varying the packet sending rate i.e. number of packets per second and number of nodes of the network.

1) Normalised Routing Overhead: The normalized routing overhead is the number of transmitted control packets for transferring a data packet from source to destination.

\section{Analysis for Routing Overhead}

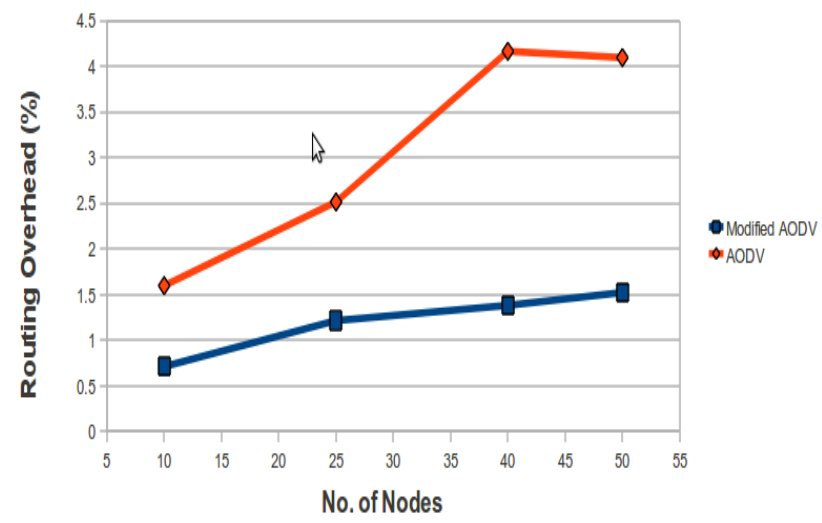

Fig. 5: Graph of routing overhead vs No. of Nodes

Fig.5 shows the graph of routing overhead verses number of nodes. It can be seen from figure that the AODV protocol required more control packets as compared with this modified AODV protocol.

But during simulation we have observed that the initial route set up overhead by this protocol is marginally higher than that of AODV because of the modified AODV (PEER) protocol requires some extra control packets at initial stage to construct the route as it examines the node's energy-factor for selecting a node in route. If the routing metric of a node is less than the threshold values, then the control packets get dropped; hence the source node re-initiates route discovery process. It can be seen that AODV try to setup the route several times by initiating the route discovery process which increases the routing overhead.

2) Energy Consumption per packet: It is measured as the total consumed energy divided by the total number of packets received.

It is clear from fig.6 that this protocol consumes lesser energy and having established route has high lifetime compared to that of AODV protocol. We found that Modified AODV (PEER) protocol is more energy efficient. 
International Journal of Advanced Research in Computer and Communication Engineering Vol. 4, Issue 5, May 2015

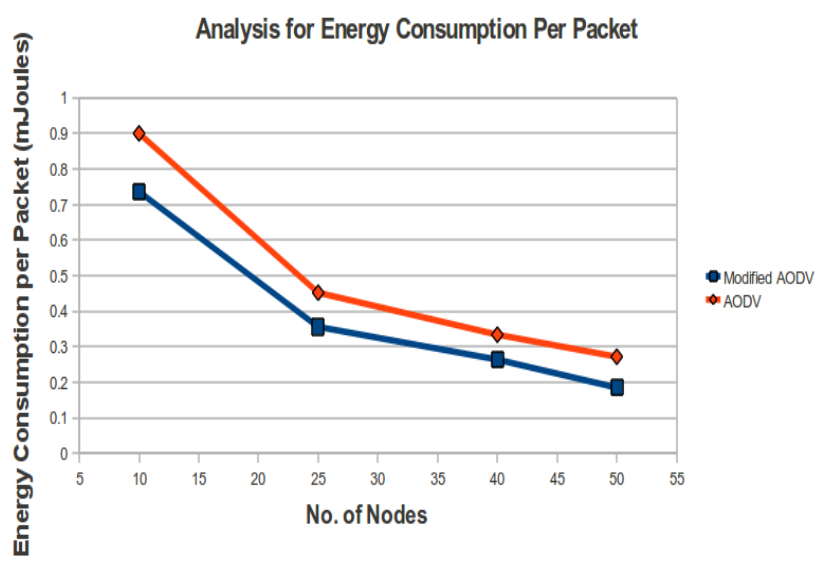

Fig. 6: Graph of Energy consumption per Packet vs No. of Nodes

From Fig 7 we found that this protocol provides a uniform energy consumption environment in MANET and thus it also increases MANET's lifetime.

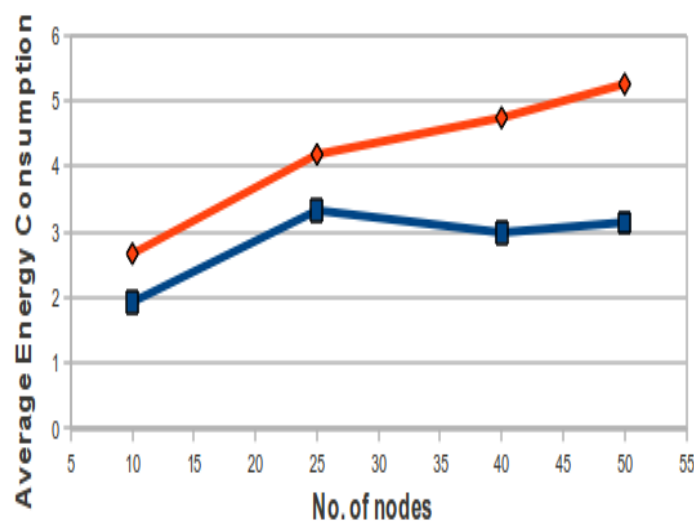

- Modfied AODV $\triangle A O D V$

Fig. 7: Graph of Energy consumption vs No. of Nodes

3) Average End to End Delay: The average time from the beginning of packet transmission at the source node until its receipt at the destination.

From Fig. 8, it can be seen that the end-to-end delay for this protocol is less as compared to AODV protocol. In this protocol, there are less chances of link failure in route constructed by AODV protocol because of power failure of node.

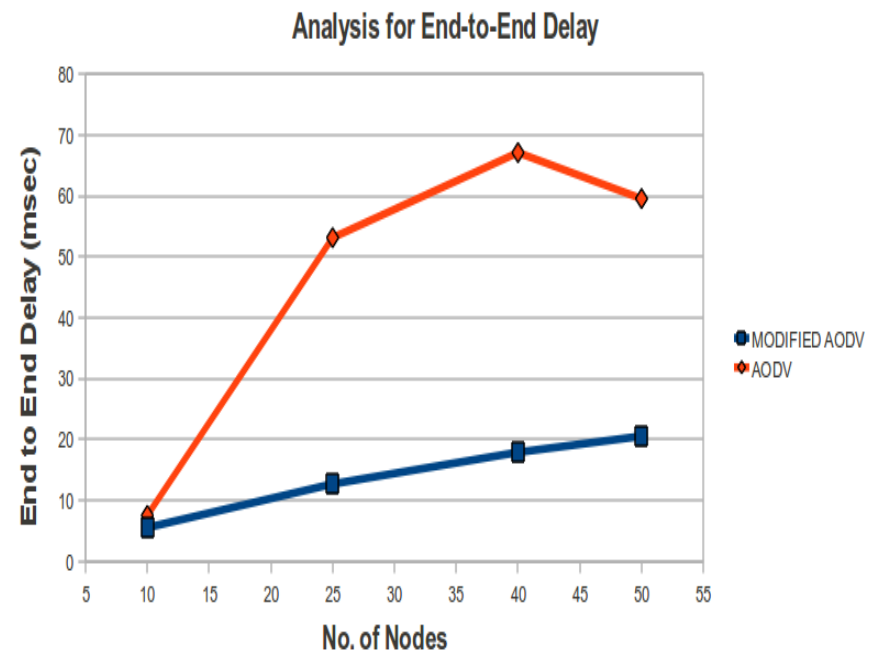

Fig. 8: Graph of End-to-End Delay vs No. of Nodes

4) Packet Delivery Ratio (PDR): It is the ratio of number of packets successfully delivered to destination to the number of packets transmitted by source node.

\section{$P D R=\underline{(\text { Total number of packets successfully received })}$} (Total number of packets sent)

Fig.9 shows the graph for PDR for different packet rate. It can been seen that in by using this protocol packets are less dropped by intermediate node due to insufficient energy and by using AODV protocol packets may get drooped due to route failure just because of insufficient energy of the node in established route.

\section{Analysis for PDR}

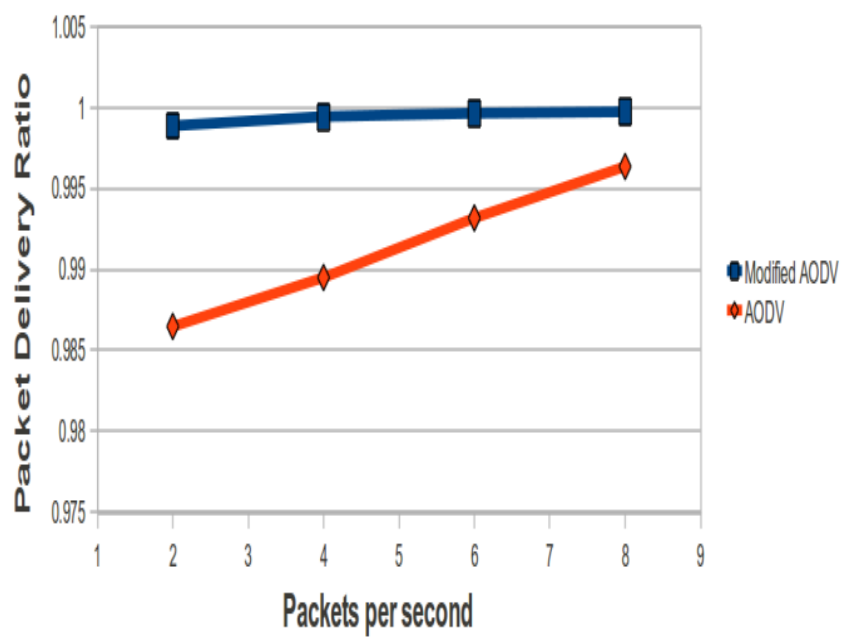

Fig. 9: Graph of Packet Delivery Ratio vs Packets per Sec. 


\section{CONCLUSION}

We have successfully implemented the energy-efficient routing into which reliable path in terms of sufficient energy having nodes are selected in the route by means of which efficiency is achieved in terms of energy consumption factor.

By implementation the proposed concept in Network Simulator-2 (NS-2) it has been observed that new proposed protocol can select better routes in which no node death is observed until communication is over. This indicates the nodes with energy with less than threshold are available for further communication where less energy requirements are and hence total network life time is enhanced. Through simulation and from the results, it can be seen that there is drastically reduction in link failures to occur in the route constructed by this protocol.

\section{FUTURE SCOPE}

In the implemented system, we have established energyefficient route in between source-destination pair. However, while dealing with multiple source and destination pair, there is a possibility of sharing a common node which is termed as mutual node. If multiple routes sharing a mutual node, so that particular mutual node may get drained faster in terms of energy consumption which again causes that node die earlier. This may deteriorate the lifetime of entire mobile ad-hoc network.

To avoid such situations and to avoid node energy run out problems due to loading on single node, its load can be shared amongst its neighbouring node. This will lead to energy conservation and will avoid node deaths due to excessive energy consumption.

In this way trust based route selection and load sharing will keep maximum number of nodes in the network live and will maximize the lifetime of entire mobile ad-hoc network.

\section{ACKNOWLEDGMENT}

First and foremost I want to thank my Guide Prof. D.G. Chougule for constant encouragement and noble guidance. With great pleasure and gratefulness, I extend my deep sense of gratitude to Prof. S. S. Patil, HOD, Electronics Engg. Deptt. for giving me an opportunity to accomplish my paper and to increase my knowledge. Lastly I wish each and every person involved in making my dissertation successful. Thank You.

\section{REFERENCES}

1] C. Yu, B. Lee, and H. Y. Youn, "Energy efficient routing protocols for mobile ad-hoc Networks," WIRELESS COMMUNICATIONS AND MOBILE COMPUTING, vol. 3, No. 8, pp. 959-973, 2003.

[2] J. M. Kim and J. W. Jang, "AODV based Energy Efficient Routing Protocol for Maximum Lifetime in MANET," AICT-ICIW, pp. 77,February 2006.

[3] D. Feng and Y. Zhu, "An Improved AODV Routing Protocol Based on Remaining Power and Fame," Electronic Computer Technology, pp. 117-121, February 2009.

[4] V. Rodoplu and T. H. Meng, "Minimum Energy Mobile Wireless Networks," IEEE Journal on Selected Areas in Communications, vol. 17, No. 8, pp. 1333-1343, August 1999.
[5] P. J. Wan, G. Calinescu, X. Y. Li, and O. Frieder, "Minimumenergy broadcasting in static ad-hoc networks," Wireless. Network. Journal, vol. 8, No. 6, pp. 607-617, November 2002.

[6] I. Park, T. H. Meng, and I. Pu, "Blocking Expanding Ring Search Algorithm for Efficient Energy Consumption in Mobile Ad-hoc Networks," IFIP WONS, pp. 191-195, January 2006.

[7 L. M. Feeney and M. Nilsson, "Investigating the Energy Consumption of a Wireless Network Interface in Ad-hoc Network Environment," IEEE INFOCOM, vol. 3, pp. 1548-1557, April 2001.

8] B. Sun, C. Gui, and P. Liu, "Energy Entropy Multipath Routing Optimization Algorithm in MANET based on GA," Proc. Fifth IEEE Int. Conf. on Bio-Inspired Computing: Theories and Applications (BICTA), pp. 943-947, September 2010

[9] A. Syarif and R. F. Sari, "Performance Analysis of AODV-UI Routing Protocol With Energy Consumption Improvement Under Mobility Models in Hybrid Ad-hoc Network," Int. Journal on Computer Science and Engineering (IJCSE), vol. 3, pp. 2904-2918, July 2011.

[10] J. Zhu and X. Wang, "Model and Protocol for Energy-Efficient Routing over Mobile Ad-hoc Network," IEEE TRANSACTIONS ON MOBILE COMPUTING, vol. 10, pp. 1546-1557, September 2011.

[11] N. Sumathi and A. S Thanamani, "Evaluation of Energy Efficient Reactive Routing Protocols in QoS Enabled Routing for MANETS," Int. Journal of Computer Applications, vol. 14, pp. 1014, January 2011

[12] E. L. Lloyd, R. Liu, M. V. Marathe, R. Ramanathan, and S. S. Ravi, "Algorithm aspects of topology control problems for ad-hoc networks," Mobile Netw. Appl., vol. 10(1-2), pp. 19-34, February 2005.

[13] V. Mohit, J. Murtuza, and C. Madhusudhanan, "Quatifying Trust in Mobile Ad-Hoc Networks," IEEE Int'l Conf. on Integration of Knowledge Intensive Multi-Agents Systems, Waltham, MA, USA, pp. 65-70, May 2005.

[14] Y. Hou, Y. Shi, J. Pan, A. Efrat, and S. Midkiff, "Maximizing the lifetime of Wireless sensor networks through single-session flow routing," IEEE Transaction, Mobile Computing., vol. 5, No. 9, pp. 1255-1266, September 2006.

[15] F. Guerreiro, F. De Rango, S. Marano, and E. Bruno, "A biobjective optimization model for routing in mobile Ad-hoc Networks," Applied Mathematics Modeling, vol. 33, pp. 1493-1512, March 2009.

[16] AODV, “Ad-hoc on-demand Distance Vector Routing”, RFC3561. 